\title{
EL ABEJARUCO (MEROPS APIASTER L., AVES: MEROPIDAE) Y EL SECTOR APÍCOLA DE LA REGIÓN DE MURCIA (SE ESPAÑA): PERCEPCIÓN DE SU IMPACTO Y MODELO ECOGEOGRÁFICO DE DISTRIBUCIÓN
}

\author{
Pablo Farinós ${ }^{l}$, Víctor M. Zapatal ${ }^{1}$ Vicente Martínez-López ${ }^{l}$, \\ $M^{a}$ Victoria Jiménez ${ }^{1}$ y Francisco Robledano ${ }^{1}$ \\ Universidad de Murcia
}

\section{RESUMEN}

Se analiza desde una perspectiva ecogeográfica el riesgo de predación del abejaruco (Merops apiaster) sobre la abeja melífera (Apis mellifera), para evaluar la percepción de su impacto sobre los colmenares por el sector apícola de la Región de Murcia. Para ello, se realizaron encuestas a apicultores y se elaboró una cartografía regional de idoneidad de hábitat a partir de modelos de distribución de nicho ecológico (ENFA), teniendo en cuenta registros de presencia obtenidos en el campo. Los resultados son compatibles con la proximidad entre colmenas y aves, lo que explica la percepción de un alto riesgo de predación. Se proponen estrategias para mitigar el posible impacto de la predación a través de la relocalización de colmenares en los hábitats y paisajes que los acogen.

Palabras clave: abejaruco, abeja melífera, ENFA, distribución, impacto.

\section{THE BEE-EATER (MEROPS APIASTER) AND THE BEEKEPING SECTOR OF MURCIA REGION (SE SPAIN): PERCEPTION OF ITS PREDATION IMPACT AND ECOGEOGRAPHICAL DISTRIBUTION MODEL}

\begin{abstract}
This paper analyzes, from an ecogeographical perspective, the risk of predation by the Bee-eater (Merops apiaster) on the honeybee (Apis mellifera), in order to assess the perception of its impact on the apiaries of the Region of Murcia. To this end, an inquiry to bee-keepers has been made, and a regional cartography of habitat suitability has been developed on the
\end{abstract}

Fecha de recepción: 21 de Julio de 2014. Fecha de aceptación: 25 de Noviembre de 2014.

1 Departamento de Ecología e Hidrología, Facultad de Biología, Campus Universitario Espinardo, Universidad de Murcia, 30100 Murcia, pfarinos@um.es 
basis of Ecological Niche Factor Analysis (ENFA) distribution models, taking into account field records of presence. Results are consistent with the proximity between beehives and birds, explaining the perception of a high risk of predation. Strategies are proposed to mitigate the potential impact of bird predation, through the relocation of apiaries in the habitats and landscapes occupied by them.

Key words: bee-eater, honeybee, ENFA, distribution, impact.

\section{INTRODUCCIÓN}

El abejaruco europeo (Merops apiaster Linnaeus, 1758) es una especie de ave perteneciente al Orden Coraciiformes, dentro del cual es el principal y único representante con presencia regular en España de la familia Meropidae. Su apariencia y comportamiento le hacen prácticamente inconfundible, con cuerpo fusiforme de $28 \mathrm{~cm}$ de longitud, pico alargado y curvo, alas y cola largas y puntiagudas y viva coloración, básicamente azulada en la parte inferior, pardo amarillenta en la superior y amarilla en el cuello. Se suele ver posado sobre cables y árboles, con frecuencia en grupos, y emite un reclamo característico y de largo alcance, principalmente en vuelo. Es una especie estrictamente insectívora con preferencia por himenópteros, y dentro de ellos principalmente abejas domésticas (Apis mellifera Linnaeus, 1758) (VALERA, 2014).

El abejaruco ha experimentado una expansión en el Mediterráneo occidental, documentada en España desde principios del S. XX y sobre todo a partir de las décadas de 1960-70 (BERNIS, 1970; MUNTANER et al., 1983). Algunos autores indican una probable relación entre el incremento de la apicultura a tiempo parcial y el gran crecimiento de la población (MASSA y RIZZO, 2002), fenómeno que también se relaciona con cambios recientes en la gestión del paisaje rural de la cuenca mediterránea, como las medidas agroambientales que han favorecido las áreas abiertas ricas en flora melífera por las que esta especie muestra preferencia (MASSA y LA MANTIA, 2007). Tampoco pueden descartarse otras causas, como la suavización de las condiciones climáticas (KINZELBACH et al., 1997).

Parece que la preocupación por el impacto de los abejarucos sobre la supervivencia de las abejas y la viabilidad de los colmenares es un fenómeno coetáneo con el aumento poblacional y la expansión de la especie, aunque recientemente se ha incrementado al conjugarse con otras causas de mortalidad y pérdida de producción (sequía, enfermedades...). Además, se extiende el temor de que una mayor presencia y expansión geográfica (facilitadas por el cambio climático), junto con un alto grado de especialización en la captura de abejas, incremente este factor de presión hasta extremos que localmente puedan resultar insostenibles.

Dado que la impresión general era que los apicultores se sentían perjudicados por la presencia y predación por parte del ave, el estudio se enfocó a conocer cuál era la percepción de éstos sobre la distribución y naturaleza de los efectos causados por el abejaruco en sus colmenares, y a establecer un modelo de distribución regional del ave basado exclusivamente en sus preferencias ecológicas. Como hipótesis de investigación se planteó que la percepción del sector apícola podría responder a una distribución del ave determinada 
por características ecogeográficas similares a las que condicionan la localización de los colmenares. Esto apoyaría la existencia de un riesgo de predación que debería ser abordado mediante diferentes estrategias (de localización espacial de los colmenares, de mitigación de los daños, o de compensación de pérdidas). En caso contrario, la opinión del sector apícola obedecería a una percepción distorsionada que podría deberse a la transmisión de información errónea, o a la atribución de daños debidos a otros factores (enfermedades, deterioro de los hábitats...).

Los objetivos establecidos son:

- Determinar, mediante consultas directas, la opinión del sector apícola acerca de la incidencia del abejaruco sobre la apicultura en la Región de Murcia (SE Ibérico), caracterizando su percepción sobre la distribución de la especie y la naturaleza e importancia de su impacto sobre los colmenares de abeja doméstica.

- Elaborar una cartografía de idoneidad del hábitat basada en datos de presencia del abejaruco obtenidos por registro directo en el campo, y en el análisis de las variables ambientales que más influyen en su ocupación del territorio.

- Proponer estrategias de actuación y posibles medidas para mitigar, en su caso, el impacto de la predación del abejaruco sobre los colmenares.

\section{2. ÁREA DE ESTUDIO Y METODOLOGÍA}

\section{1. Área de estudio}

El área de estudio incluye todo el territorio de la Comunidad Autónoma de la Región de Murcia, situada en el sureste de la Península ibérica, entre los $37^{\circ} 22^{\prime}$ y $38^{\circ} 45^{\prime}$ latitud Norte y los $0,43^{\prime}$ y $12^{\circ} 20^{\prime}$ de longitud Este. Abarca una superficie de $11.317 \mathrm{~km}^{2}$ de la cual el 23\% se halla a una altitud inferior a $200 \mathrm{~m}$ y el 32,3\% por encima de los $600 \mathrm{~m}$. El clima es mediterráneo semiárido, con veranos cálidos y secos e inviernos suaves. La precipitación anual se sitúa entre 300-350 mm/año con variaciones territoriales y distribución irregular, concentrándose en primavera y otoño. La temperatura media anual oscila entre los 15 y $19^{\circ} \mathrm{C}$ variando según la altitud. Constituye un verdadero ecotono biogeográfico entre la Cuenca Mediterránea y los desiertos sub-tropicales del sur, albergando una notable biodiversidad (CALVO et al., 2000), favorecida por su elevada heterogeneidad física (geológica, topográfica, geomorfológica, edáfica, etc.).

\subsection{Consulta al sector apícola}

Durante 2008, se realizaron encuestas telefónicas para conocer la opinión del sector apícola sobre la incidencia del abejaruco. La encuesta se enfocó en conocer cómo perciben los apicultores los efectos causados por el abejaruco en sus colmenares, así como en obtener datos adicionales acerca de la presencia de la especie en la Región de Murcia. La consulta se pudo realizar gracias a los datos telefónicos proporcionados por la Asociación de Apicultores de la Región de Murcia (AARM). Inicialmente, se anotaban en la hoja de respuestas los datos personales del apicultor (nombre, localidad, teléfono y domicilio). A 
continuación, se procedía a realizar la encuesta, que constaba de 12 preguntas, la primera de ellas de localización y caracterización del colmenar, y el resto sobre la presencia e impacto del abejaruco:

1. Número de colmenas que componen el colmenar: además, se solicitaba información lo más precisa posible de situación, como el paraje, la localidad, el término municipal y las coordenadas UTM (dato que no pudo ser proporcionado por ningún apicultor). También se preguntaba por el periodo de presencia de los colmenares en sus localizaciones (si eran fijos o trashumantes).

2. Se observan abejarucos en las proximidades: en el caso de que la respuesta fuera negativa, se daba por finalizada la encuesta.

3. En caso afirmativo, se dividió la presencia en tres categorías: escasa, abundante o muy abundante.

4. $\mathrm{N}^{\mathrm{o}}$ (aproximado) de abejarucos observados por el apicultor en sus colmenas (4 categorías): menos de 10, menos de 50, menos de 100 o más de 100.

5. En qué fechas principalmente eran observados (4 categorías): Marzo-Mayo, JunioJulio, Agosto-Septiembre y Otras (en este último caso, fechas anteriores a marzo o posteriores a septiembre). Algunos indicaron que si el invierno no era muy frío, podían permanecer todo el año (lo cual parece muy improbable).

6. ¿Existen nidos o colonias de estas aves cerca del colmenar? Si la respuesta era afirmativa, se intentaba que los apicultores estimaran a qué distancia aproximada de las colmenas se encontraban. A veces, los apicultores no habían visto ese año abejarucos en los nidos, pero si conocían lugares de hábitat favorable para la nidificación cercanos a las colmenas.

7. Daños directos que el abejaruco provoca en su colmenar: no se producen, escasos, moderados o considerables.

8. Valore de 1 a 10 donde considera que se producen los principales daños: esta valoración resultaba difícil, por lo que se pasó a preguntar a qué nivel (o en qué segmento) de la organización social de la colmena se manifestaban los mayores daños, pudiendo corresponder a la pérdida de abejas obreras, la pérdida de enjambres, la pérdida de colmenas completas o la captura de la reina.

9. ¿Existen posaderos para las aves próximos al colmenar (cables, postes, árboles...)? Si o no. Con el transcurso de las encuestas, los apicultores fueron quitando importancia a su presencia, porque afirmaban ver a los abejarucos posados incluso encima de las propias colmenas.

10. ¿Qué porcentaje de colmenas considera que ha perdido por causa del abejaruco? Más que una pérdida directa de colmenas, las respuestas reflejaban un debilitamiento de las mismas por la predación de abejas obreras, lo que a medio-largo plazo podía mermar la capacidad de la colmena para mantenerse. Tan sólo se podía atribuir directamente al abejaruco la pérdida de colmenas debida a la predación de la abeja reina. No obstante, algunos apicultores fueron capaces de proporcionar un porcentaje de pérdida de colmenas.

11. ¿En qué porcentaje estima que reduce el abejaruco la producción anual de miel del colmenar? Como la anterior, esta pregunta fue complicada de responder, aunque 
muchos apicultores proporcionaron directamente datos porcentuales de reducción de la producción. Otros recordaban la cantidad de miel producida en años con distinta incidencia del abejaruco, lo que permitía estimarla.

12. ¿Ha perdido enjambres o colmenas por otras causas (enfermedades...)? pretendía recabar información sobre otras causas de debilitamiento o mortalidad.

Por último, existía un apartado de observaciones donde se registraba cualquier dato adicional aportado que pudiera ser relevante para los objetivos del estudio. Además, se preguntaba al apicultor sobre su disposición a volver a colaborar si fuera necesario.

\subsection{Muestreo de campo}

Para la obtención de datos directos de presencia en el campo se realizaron recorridos en vehículo orientados a cubrir los principales gradientes geográficos y ambientales de la Región de Murcia. Este tipo de metodología ha sido aplicado a otras especies de aves de fácil detectabilidad (TELLERÍA, 1986; SUNYER y VIÑUELA, 1996). Se siguió el criterio de evitar carreteras muy transitadas y áreas homogéneas (en cuando a altitud, relieve, usos del suelo y otras características ecogeográficas generales).

\section{FIGURA 1}

Principales recorridos de prospección realizados durante la primavera-verano de 2008, cubriendo de forma sistemática los principales gradientes geográficos y climáticos de la región. Estos itinerarios se complementaron con otros de menor longitud para cubrir áreas específicas o completar los principales ejes de prospección.

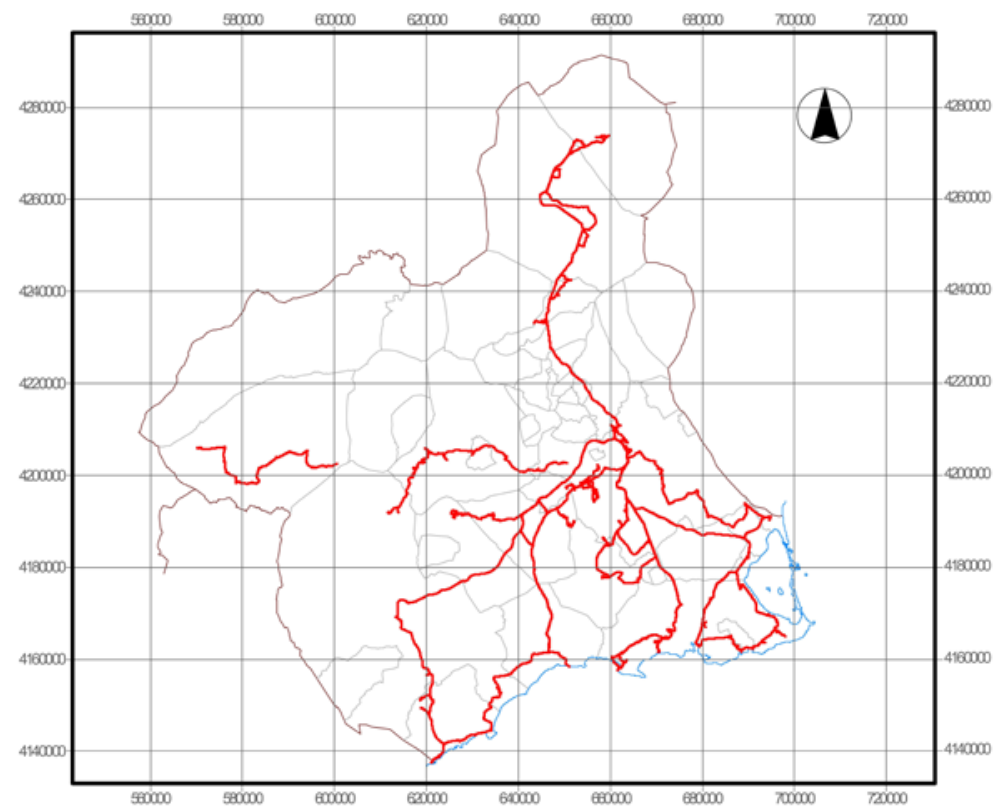


Los itinerarios y puntos de observación se registraron mediante GPS (las rutas aparecen reflejadas en la Figura 1). Se recorrieron durante la primavera y verano de 2008, realizando recorridos de una longitud media de $98,98 \mathrm{~km}(\mathrm{~N}=15$, rango 25,43-243,6), para cubrir una longitud total de $1.484,8 \mathrm{~km}$. El vehículo circulaba a una velocidad moderada (27,56 km/h de media), con 2-3 observadores permanentemente atentos a la detección de aves posadas en las cercanías de la ruta. Además, se realizaban paradas regulares (aproximadamente cada km si antes no se producía ninguna observación), en especial cerca de hábitats potenciales de nidificación (ramblas, desmontes...), para emitir el reclamo del ave durante un máximo de 10' (si antes no se obtenía respuesta de un ave cercana). El uso de carreteras de nivel superior resultaba desaconsejable, ya que habría impedido progresar a la velocidad deseada y realizar paradas, y el ruido de fondo habría dificultado la audición del reclamo. Dada la naturaleza del análisis utilizado para modelizar la distribución, que se describe en el apartado siguiente, no se requerían datos de abundancia ni un esfuerzo uniforme de prospección. Por ello, el trabajo de campo se orientó a obtener registros de presencia de la especie dentro de cuadrículas UTM de 1x1 km, en un rango lo más amplio posible de variabilidad ambiental, y en ningún caso a cuantificar el número de individuos observados por tramo recorrido o punto de parada. Para incrementar el tamaño de muestra, el modelo de distribución fue finalmente construido con los registros de presencia obtenidos directamente en el campo por el equipo investigador, junto con los registros equivalentes facilitados por colaboradores externos expertos que realizaron prospecciones similares durante el periodo de estudio.

\subsection{Modelos de distribución y cartografía de potencialidad del hábitat}

La falta de información sobre las áreas en las cuáles el abejaruco está efectivamente ausente (frente a aquéllas en las que su presencia no ha sido detectada durante el muestreo) dificulta la utilización de las herramientas comunes de modelización ecológica, basadas en datos de presencia/ausencia (SANTOS et al., 2006). Por este motivo, se hace necesario utilizar una técnica de modelización que sin precisar la ausencia, permite identificar los factores ambientales que explican la presencia de la especie, así como las áreas de hábitat potencial o favorable.

Con los datos de presencia de abejaruco recopilados (92 presencias) se elaboraró un modelo de distribución regional mediante un Análisis Factorial de Nicho Ecológico o ENFA (Ecological Niche Factor Analysis) utilizando el software Biomapper 4.0. Los ENFA (HIRZEL et al., 2002) se basan en comparar las distribuciones de diversas variables ecogeográficas (EGVs) entre el conjunto de datos de presencia y la totalidad del área de estudio (en este caso la Región de Murcia). El ENFA resume las EGVs seleccionadas por los investigadores (por considerarlas relevantes para la ecología de las especies) en unos pocos factores no correlacionados que retienen la mayor parte de la información. El primer factor, llamado marginalidad, describe la distancia entre el «hábitat óptimo» de la especie y el «hábitat medio» en el área de estudio, es decir, la dirección en la cual el nicho de la especie se diferencia más de las condiciones disponibles (SANTOS et al., 2006). Los coeficientes (scores) de la matriz de marginalidad indican la correlación entre cada variable ecogeográfica y este factor. Cuanto mayor es el valor absoluto, mayor es 
su contribución a dicho factor. Un valor cercano a cero indica que la especie vive en las condiciones promedio del área de estudio, mientras que un valor próximo a uno indica una alta selectividad (o la tendencia a vivir en hábitats extremos). Un valor positivo significa que la especie muestra preferencia por valores altos en una determinada característica o variable ecogeográfica (por ejemplo, la presencia de un determinado sustrato o tipo de vegetación), mientras que un valor negativo indica una baja preferencia o rechazo. Las EGVs utilizadas fueron 6 clases litológicas, la altitud, la abruptosidad y una reclasificación en 8 tipos de usos del suelo. Se obtuvo además un mapa regional de idoneidad de hábitat que clasifica el territorio en función de la preferencia de uso de la especie.

\section{RESULTADOS}

\subsection{Opinión del sector apícola}

Los resultados se presentan con una estructura similar a la del cuestionario utilizado, exponiendo y comentando para cada pregunta los porcentajes de respuesta.

\subsubsection{Tamaño y distribución geográfica de la muestra}

El número de apicultores cuyos datos fueron suministrados por la Asociación de Apicultores de la Región de Murcia, fue de 163, de los cuales 75 (46,01\%) fueron consultados mediante encuesta telefónica, accediendo a realizar la encuesta el 100\% de los mismos. Los encuestados se distribuían por 28 municipios (algo más del 60\% de los de la región) con una media de 2,74 apicultores por término municipal (rango 1-10), siendo los municipios con mayor número de ellos Cehegín (10), Murcia (9) y Caravaca, Calasparra, Moratalla y Pliego (5), y Puerto Lumbreras (4). En el resto sólo fueron encuestados 1-2 apicultores. En el sector apícola regional, la trashumancia predomina sobre la permanencia $(55,86 \%$ frente a 38,62\% de los apicultores encuestados que respondieron a esta cuestión), y dependiendo del lugar de destino, los daños que puede provocar el abejaruco son muy distintos. La dominancia de la trashumancia sobre la permanencia hace que los daños que puede provocar el abejaruco sean mayores en aquellas colmenas que trashuman para ayudar en la polinización de ciertos cultivos y que suelen situarse en zonas más propicias para la presencia del ave (zonas de baja altitud cercanas a cauces fluviales, paisajes agrarios o agroforestales abiertos).

\subsubsection{Percepción de la presencia y abundancia}

De los apicultores encuestados (75), el 91,89\% (69) afirma haber observado el ave en sus colmenares, y el $8,11 \%$ (6) no. En la Tabla 1 se muestran los resultados de las encuestas relativos a las estimas cualitativas y cuantitativas de presencia del abejaruco en el entorno de las colmenas.

Cabe destacar que el $94,12 \%$ de los colmenares poseen posaderos para el ave (cables, postes, árboles,...), pero según las respuestas de los apicultores, esto no es un problema para el abejaruco pudiendo posarse directamente sobre las colmenas o realizar sus ataques en vuelo. Aun así, la presencia se percibe de diferente manera dependiendo del término 
municipal, tanto cualitativa como cuantitativamente. Existen municipios donde la presencia se señala como «abundante» y dicen observarlos en bandadas de más de 100 individuos, y otros donde la califican de «muy abundante» y tan sólo se observan de media entre 10-50 individuos (datos no mostrados). De todos modos, la percepción general de muchos apicultores fue que el año 2008 había sido un año atípico, observándose el ave en abundancias muy inferiores a años anteriores (lo cual contrasta con las elevadas cifras declaradas por ellos). Esta impresión tampoco concuerda con la tendencia estimada para la especie en programas de seguimiento científico: el abejaruco muestra una tendencia catalogada como «estable» según los resultados del programa SACRE para el periodo 1998-2013 (ESCANDELL, 2014).

\section{TABLA 1}

Estimas cualitativas y cuantitativas de la abundancia del abejaruco en el entorno de las colmenas de los apicultores encuestados ( $\mathrm{N}=75$ en ambos casos)

\begin{tabular}{|c|c|c|c|}
\hline Estima cualitativa & Abundancia & $\mathrm{N}^{\circ}$ de apicultores & $(\%)$ \\
\cline { 2 - 4 } & Escasa & 10 & 13,24 \\
\cline { 2 - 4 } & Abundante & 32 & 42,65 \\
\cline { 2 - 4 } & Muy abundante & 33 & 44,12 \\
\hline Estima cuantitativa & $\mathrm{N}^{\mathrm{o}}$ de aves observadas & $\mathrm{N}^{\circ}$ de apicultores & $(\%)$ \\
\cline { 2 - 4 } & $<10$ & 6 & 7,53 \\
\cline { 2 - 4 } & $10-50$ & 32 & 42,65 \\
\cline { 2 - 4 } & $51-100$ & 17 & 23,53 \\
\cline { 2 - 4 } & $>100$ & 20 & 26,47 \\
\hline
\end{tabular}

El 57,58\% de los apicultores encuestados conocían la existencia de nidos de abejaruco cerca de sus colmenares, mientras que el 42,42\% restante lo desconocía. La distancia entre los nidos y las colmenas varía desde $20 \mathrm{~m}$ a $5 \mathrm{~km}$, obteniendo valores medios entre 500-700 $\mathrm{m}$ de distancia.

\subsubsection{Percepción de daños}

En la tabla 2 se muestran los datos obtenidos en las encuestas sobre el nivel de daño que produce el abejaruco a las colmenas de los apicultores que detectaron el ave en su entorno.

\section{TABLA 2.}

Nivel de daño estimado sobre las colmenas según la opinión de los apicultores encuestados $(\mathrm{N}=75)$

\begin{tabular}{|c|c|c|}
\hline Daños estimados & $\mathrm{N}^{\mathrm{o}}$ de apicultores & $(\%)$ \\
\hline No provoca & 2 & 2,94 \\
\hline Escasos & 4 & 5,88 \\
\hline Moderados & 19 & 25 \\
\hline Considerables & 50 & 66,18 \\
\hline
\end{tabular}


El 59,00\% de los apicultores opina que la mayor presión se da sobre las abejas obreras produciendo un importante debilitamiento de la colmena. Un 6,00\% opina que el mayor estrés se da a nivel de enjambre, y un $12,00 \%$ a nivel de colmena. Cabe destacar la presión atribuida a la captura de las abejas reinas durante su vuelo nupcial, que aunque no sea mencionado por los apicultores con un porcentaje alto $(23,00 \%)$, es de gran importancia puesto que su desaparición se traduce en la pérdida de la colmena.

Aun así, un 52,54\% de los apicultores encuestados han sido capaces de contestar a la pregunta: ¿qué porcentaje de colmenas son perdidas a causa del abejaruco) (Tabla 3).

Sobre la reducción de la cosecha miel por causa del abejaruco, los porcentajes de respuesta son inferiores (sólo un 39,71\% de los encuestados; Tabla 3).

\section{TABLA 3}

Opinión de los apicultores encuestados sobre las pérdidas de colmenas ( N=39) y reducción de la cosecha de miel $(\mathrm{N}=31)$

\begin{tabular}{|c|c|c|c|c|}
\cline { 2 - 5 } \multicolumn{1}{c|}{} & \multicolumn{2}{c|}{ Pérdida de colmenas } & \multicolumn{2}{c|}{ Reducción en la cosecha de miel } \\
\cline { 2 - 5 } \multicolumn{1}{c|}{} & Número & Porcentaje & Número & Porcentaje \\
\hline$<10 \%$ & 2 & 5,56 & 2 & 7,41 \\
\hline $10-19 \%$ & 12 & 30,56 & 8 & 25,93 \\
\hline $20-29 \%$ & 11 & 27,78 & 7 & 22,22 \\
\hline $30-49 \%$ & 8 & 19,44 & 7 & 22,22 \\
\hline$>49 \%$ & 6 & 16,67 & 7 & 22,22 \\
\hline
\end{tabular}

Sin embargo, aunque no ha sido posible cuantificar el porcentaje real de pérdida debido a otros factores, parece claro que existen otras causas de mortalidad de abejas (enfermedades como la varroa o el pollo escayolado, comentadas al margen de la encuesta por algunos apicultores consultados), que tendrían efectos importantes sobre la viabilidad o rentabilidad de los colmenares.

\subsection{Modelo de distribución y variables ecogeográficas relacionadas}

El modelo de distribución fue finalmente construido con los registros de presencia en 92 cuadrículas UTM de 1x1, la mayoría (56) obtenidos directamente en el campo por el equipo investigador, y el resto (36) facilitados por colaboradores externos confiables durante el año 2008. En el modelo (Figura 2), el abejaruco prefiere litología cuaternarias y margosas, a priori los materiales más fácilmente excavables, pero también los que suelen ocupar terrenos de menor altitud, una de las variables que más claramente evita (marginalidades de $-0,43$ a $-0,59$ ), junto con la abruptosidad, también con valores negativos relativamente altos, entre $-0,25$ y $-0,33$ (Tabla 4). Esto confirma su preferencia por terrenos de naturaleza sedimentaria, de escasa pendiente (valores máximos del $40 \%$ ) y baja altitud (ZAPATA, 2008).

También constituyen áreas de baja idoneidad las correspondientes a litologías metamórficas (marginalidad negativa siempre superior a 0,4), de conglomerados y glacis, así como las calizas. La evitación de estos materiales concuerda con su carácter coherente 
y más difícilmente excavable, lo que con carácter general los hace inapropiados para la nidificación. También está de acuerdo con su mayor frecuencia de aparición en zonas de mayor altitud donde la presencia del abejaruco se torna más rara.

\section{TABLA 4}

Valores de marginalidad del modelo de idoneidad del hábitat generados sobre la base de los datos de presencia obtenidos por el equipo investigador. Los colores amarillo y rojo (o blanco y gris) indican marginalidad positiva (máxima en el primer caso) y el azul y negro (o tonos más oscuros) marginalidad negativa (máxima en el segundo caso). Para las variables cuya correlación no es significativa no se muestra color.

\begin{tabular}{|l|c|}
\cline { 2 - 2 } \multicolumn{1}{l|}{} & Marginalidad \\
\hline LITOLOGÍA: & $\mathbf{- 0 , 4 3 1}$ \\
\hline 1. METAMÓRFICO & $\mathbf{- 0 , 1 9 6}$ \\
\hline 2. CALIZAS & $\mathbf{0 , 3 9 9}$ \\
\hline 3. MARGAS & $\mathbf{- 0 , 1 5 9}$ \\
\hline 4. CONGLOMERADOS & $\mathbf{0 , 2 7 1}$ \\
\hline 5. CUATERNARIO & $\mathbf{- 0 , 0 7 9}$ \\
\hline 6. GLACIS & $\mathbf{- 0 , 4 3 1}$ \\
\hline ALTITUD & $\mathbf{- 0 , 3 3 4}$ \\
\hline ABRUPTOSIDAD & \\
\hline USOS: & $\mathbf{0 , 1 5 7}$ \\
\hline 1. ACTIVIDADES HUMANAS & $\mathbf{0 , 2 0 4}$ \\
\hline 2. SECANO & $\mathbf{0 , 1 0 2}$ \\
\hline 3. REGADÍO & $\mathbf{- 0 , 1 8 5}$ \\
\hline 4. BOSQUE & $\mathbf{- 0 , 2 7}$ \\
\hline 5. MATORRAL & $\mathbf{- 0 , 1 5 8}$ \\
\hline 6. HUMEDALES & $\mathbf{0 , 0 6 1}$ \\
\hline 7. RAMBLAS Y RÍOS & $\mathbf{- 0 , 0 4}$ \\
\hline 8. ACTIVIDADES MINERAS & \\
\hline
\end{tabular}

En cuanto a los usos del suelo, el modelo muestra la baja preferencia o escasa idoneidad de las zonas forestales (bosque y matorral) como hábitat potencial del abejaruco (marginalidades con valores negativos en torno a 2 ó ligeramente superiores). En cambio, las zonas humanizadas son en general zonas apropiadas u óptimas, y también los cultivos de secano. La escasa preferencia por zonas forestales tendría bastante que ver con la preferencia del abejaruco por zonas poco abruptas y paisajes descubiertos de baja altitud, apropiados tanto para la nidificación como para la captura de presas. 
Figura 2

Mapa de idoneidad de hábitat en los que se clasifica el territorio de la Región de Murcia en función de la preferencia de uso del abejaruco, basados en los registros de campo obtenidos directamente por el equipo investigador o por colaboradores externos confiables durante el año 2008 (92 presencias).

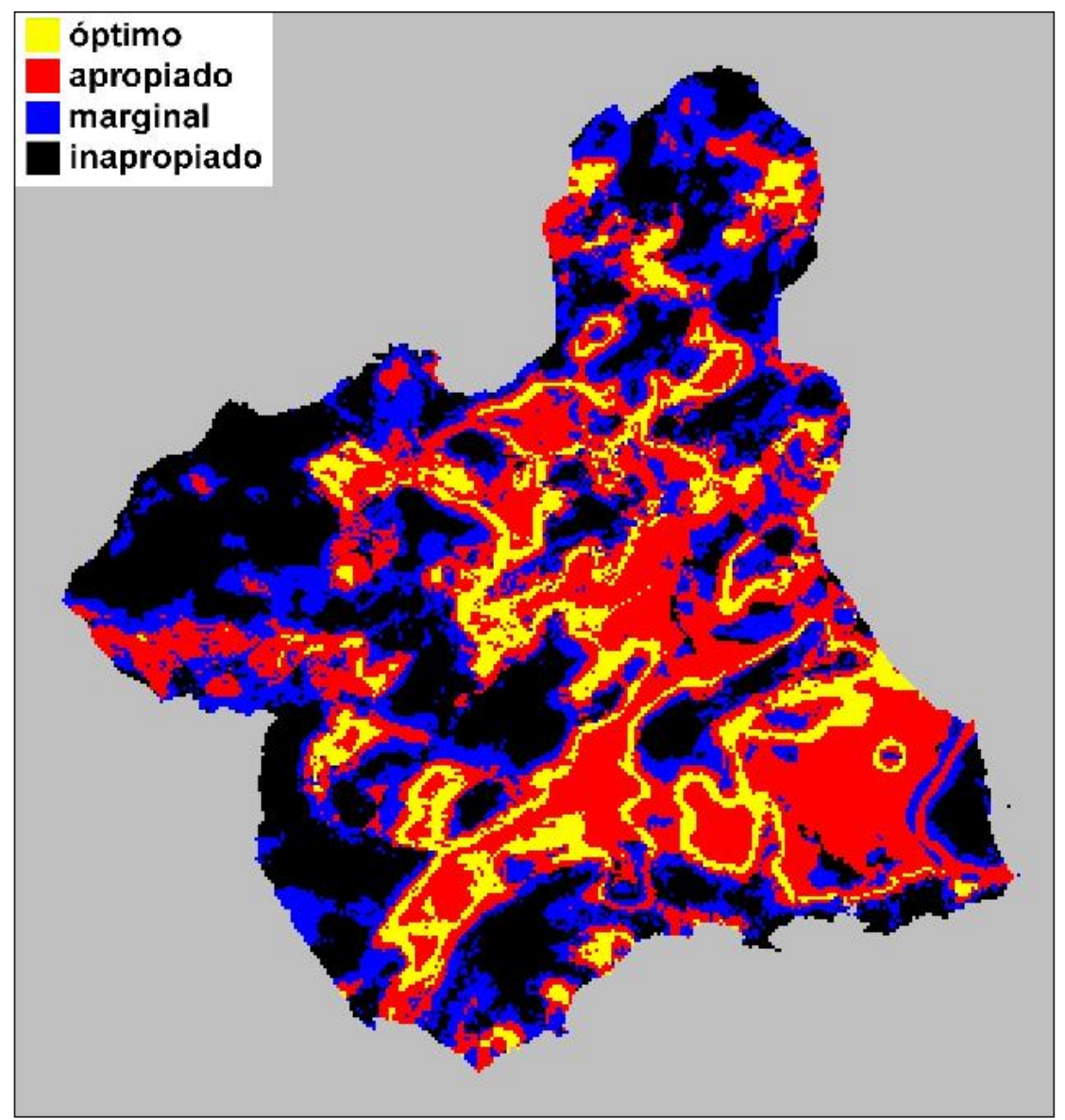

\section{DISCUSIÓN}

A la escala del presente trabajo, no se puede desmentir la incidencia percibida por los apicultores, ya que los colmenares se suelen localizar en zonas que son idóneas para el abejaruco, cuya visibilidad explicaría en muchos casos el patrón de respuesta en las encuestas, con independencia del impacto real. Investigaciones complementarias a la que aquí se presenta (ZAPATA et al., 2008; ROBLEDANO et al., 2009), demuestran que la coexistencia de abejarucos y colmenares es común, pero que el impacto real de la predación sobre las abejas obreras es, salvo situaciones puntuales, muy bajo, representando 
una parte casi inapreciable de su mortalidad. La captura de reinas, considerada importante por un porcentaje relativamente alto de apicultores, es un evento muy raro (nunca registrado después de muchas horas de observación). Esta discrepancia entre la percepción del impacto y su cuantificación real, se explicaría por un conjunto de factores. Primero, una tendencia a la sobrevaloración del impacto del abejaruco, al presentar su predación (normalmente irrelevante en el marco de la mortalidad natural de las abejas), como una sinergia negativa persistente, en combinación con otras causas objetivas de debilitación o deterioro de los colmenares (parásitos, enfermedades, insectos competidores o predadores, pesticidas, pérdida de hábitat). Esta consideración a menudo se acompaña de aseveraciones carentes de base científica o evidencia técnica, como supuestos incrementos poblacionales -desmentidos por los programas de seguimiento ornitológico (ESCANDELL, 2014)-, o comportamientos nunca observados (sedentarismo). Comparado con otras causas mucho menos notorias, la visibilidad del abejaruco hace fácil equiparar su mera presencia a impacto, lo cual seguramente hacen de forma inconsciente muchos apicultores. Una prueba de que esta atribución es más percibida que real, es la dificultad creciente que tienen los apicultores encuestados para dar respuestas precisas al tipo y magnitud de los daños ocasionados por el ave (apartado 3.1.3), o las incongruencias detectadas entre la evaluación cualitativa y cuantitativa de su presencia (apartado 3.1.2). Con la dificultad añadida de que, al ser el abejaruco una especie protegida, no le son aplicables medidas de control, tratamiento o regulación que sí pueden ser adoptadas frente a contaminantes, patógenos o parásitos. Pese al consenso de que estas últimas son las principales causas objetivas de pérdida de colonias de abejas (RATNIEKS y CARRECK, 2010), mantener al ave entre los enemigos del sector apícola contribuye a crear un clima favorable a la demanda de soluciones a las administraciones competentes por su mala situación general.

Aún así, la predación podría ser importante en épocas concretas (periodo pre-migratorio), tal como se desprende de nuestras propias observaciones (ROBLEDANO et al., 2009), o en localizaciones puntuales muy cercanas a lugares de nidificación de abejarucos (GALEOTTI e INGLISA, 2001; CHIROSA y ORANTES, 2003). En el caso de apicultores que señalan la presencia de colonias de nidificación muy próximas $(20 \mathrm{~m})$, el impacto declarado podría ser real. El abejaruco tiende a aprovechar presas de pequeño tamaño, cuando se presentan próximas y en número importante, para ahorrar energía en los desplazamientos (KREBS y AVERY, 1985), en especial durante el periodo de ceba de los pollos (SÁNCHEZ-GUZMÁN, 2007). Por ello una estrategia de evitación del riesgo de predación sería conveniente en tales casos. El fomento de las poblaciones de himenópteros silvestres en las proximidades de las colonias de nidificación de abejaruco parece también una estrategia recomendable, ya que los abejarucos concentran su alimentación en ellas por las razones arriba apuntadas (ROBLEDANO et al., 2009).

El modelo de idoneidad de hábitat (Figura 2) indica que la distribución del abejaruco se extiende por la mayor parte de la Región de Murcia, si bien existen notables diferencias en cuanto a la potencialidad del hábitat para esta especie, cuyos requerimientos le asocian preferentemente a terrenos de naturaleza sedimentaria, con pendientes poco acusadas (máximas del 40\%) y altitudes bajas, paisajes agrícolas o a lo sumo agroforestales de transición hacia los espacios de media o alta montaña. Esto concuerda con las preferencias generales atribuidas a la especie en otras regiones, donde la reproducción del abejaruco se realiza 
principalmente en terrenos forestales desarbolados y agrícolas (CHIROSA y ORANTES, 2003). Tales preferencias generan un modelo de distribución bastante parcheado, identificando como áreas más favorables determinadas orlas, corredores y depresiones, a caballo entre las zonas más intensamente aprovechadas (para la agricultura y urbanización) y el núcleo forestal de las sierras, lo que dificulta realizar una sectorización en áreas homogéneas en cuanto al riesgo de predación. La posibilidad de que los abejarucos, incluso dentro de paisajes aparentemente desfavorables por su elevación, pendiente, litología, geomorfología o uso, encuentren localmente hábitats favorables para reproducirse o alimentarse (desmontes, eriales, enclavados agrícolas, balsas de riego...), eleva el riesgo de predación de colmenares al posibilitar la proximidad espacial entre éstos y las aves. Todo lo cual complica aún más la posibilidad de establecer esa zonificación operativa, y aplicar estrategias o medidas para mitigar el conflicto entre el ave y el sector apícola.

Conjugando las dos facetas del estudio, una de las opciones para evitar la predación sería desplazar los colmenares a zonas de baja idoneidad de hábitat situadas en terrenos más elevados y de carácter predominantemente forestal, lo que implicaría aproximarlos a los espacios naturales protegidos, actualmente evitados en gran medida o utilizados marginalmente por el abejaruco. Según las citas recopiladas en el marco de esta investigación (ROBLEDANO et al., 2009), el mayor grado de solapamiento de las observaciones de abejaruco se da con algunos espacios protegidos litorales como la Sierra de la Muela-Cabo Tiñoso, y algunos LICs y ZEPA, sobre todo del Noroeste y Este de la Región. El resto de las citas, tal como predice el modelo de idoneidad del hábitat, se ajustan a una distribución en orlas periféricas a los espacios interiores de montaña, o a lo largo de corredores y valles fluviales, tanto de los principales ríos (Segura, Guadalentín) como de las numerosas ramblas que constituyen la mayor parte de la red hidrográfica de la región.

Para los colmenares «residentes», que no son desplazados por sus titulares al finalizar la temporada principal de floración de cultivos específicos (por ejemplo, azahar), podría ser recomendable facilitar su integración en los espacios naturales protegidos. Así no sólo podrían reducir su exposición a la predación, sino que ejercerían funciones beneficiosas para la flora y el paisaje natural, eventualmente financiables a través de líneas específicas de ayuda que podrían compensar los posibles daños.

\section{CONCLUSIONES Y RECOMENDACIONES}

Las principales conclusiones y recomendaciones derivadas de las consultas realizadas a los apicultores son las siguientes:

- La distribución espacial y temporal de la apicultura en la Región de Murcia es muy variable, destacando ciertos municipios por el número de colmenares y apicultores (T.M. de Murcia y noroeste de la región). La dominancia de la trashumancia sobre la permanencia hace que los daños que puede provocar el abejaruco sean variables y dependientes del destino (proximidad a cultivos).

- El daño atribuido por los apicultores al abejaruco generalmente es considerable, relacionándose sobre todo con un debilitamiento de la colmena por pérdida de abejas obreras, lo que la hace más sensible a otras amenazas. Las evidencias disponibles 
sobre predación de obreras no son coherentes con esta impresión, siendo plausible que otros agentes menos notorios (parásitos, patógenos, alteración de hábitats) sean los principales responsables directos de dicho debilitamiento.

- En general, la tendencia a mantener al abejaruco entre el catálogo de enemigos del sector apícola respondería más a su visibilidad que a la demostración de daños generalizados, aunque se sabe que puntualmente puede causarlos.

- Como herramienta para la concesión de ayudas compensatorias, sería recomendable establecer una zonificación geográfica del impacto a partir de datos de presencia real combinados con otros relativos al tipo de paisaje en que se localizan las colmenas, condicionando la concesión de ayudas al patrón de localización y desplazamiento del colmenar a lo largo del año.

- Además de compensar la predación, se podría incentivar económicamente el traslado de colmenas a zonas potencialmente menos problemáticas, como las forestales protegidas (montes públicos o espacios naturales), subvencionando los servicios ambientales que les aportan las abejas (polinización).

A éstas se añadirían las derivadas de la cartografía de idoneidad de hábitat, en relación con los requerimientos del ave, que son:

- El análisis factorial de nicho ecológico produce una distribución parcheada de las áreas óptimas (orlas, bandas de transición, corredores y depresiones correspondientes con los paisajes más favorables por su litología, topografía y vegetación).

- Esto dificulta considerablemente realizar una sectorización en grandes áreas homogéneas en cuanto al riesgo de predación, complicada además por la posibilidad de que los abejarucos, incluso en paisajes aparentemente desfavorables, encuentren localmente hábitats adecuados.

- A escala local, las características ambientales que pueden incrementar el riesgo de predación son las que favorecen el establecimiento de colonias de abejaruco, tanto las litologías fácilmente excavables, como la presencia de elementos paisajísticos que favorezcan la alimentación (cultivos, eriales, balsas de riego). Los paisajes naturales bien conservados cubiertos de vegetación son áreas poco favorables para la especie. También muestra una escasa preferencia por paisajes abruptos o en altura.

- Podría ser recomendable facilitar la integración de los colmenares «residentes» en los espacios naturales protegidos, donde no sólo reducirían su exposición a la predación (por la mayor presencia de arbolado), sino que ejercerían funciones beneficiosas para la flora y el paisaje natural.

\section{AGRADECIMIENTOS}

Los autores agradecen el apoyo técnico de la Asociación de Apicultores de la Región de Murcia (AARM) y COAG-IR (en especial por Joaquín Lozano y Carlos Zafra), y el asesoramiento de José F. Calvo y María Abellán en la elaboración e interpretación de los ENFA, así como la colaboración de Carmen García Frago de la Consejería de Agricultura y Agua de la Región de Murcia, institución financiadora de la investigación. 


\section{BIBLIOGRAFÍA}

BERNIS, F. (1970): Aves Migradoras Ibéricas. Fascículo 6. Sociedad Española de Ornitología, Madrid.

CALVO, J.F.; ESTEVE, M.A. y LÓPEZ-BERMÚDEZ, F. (Coord.). (2000): Biodiversidad. Contribución a su conocimiento y conservación en la Región de Murcia. Servicio de Publicaciones de la Universidad de Murcia, Murcia.

CHIROSA, M. (Coord.); ORANTES F.J. (2003): Estudio de la incidencia del abejaruco (Merops apiaster) en los colmenares de la Comunidad Autónoma andaluza. Consejería de Medio Ambiente, Junta de Andalucía.

ESCANDELL, V. (2014): «Programa SACRE», en: Programas de seguimiento de SEO/ Birdlife 2013. SEO/Birdlife, Madrid, pp. 4-9.

GALEOTTI, P.; INGLISA, M. (2001): «Estimating predation impact on honeybees Apis mellifera L. by European Bee-eater Merops apiaster L.». Revue d'Ecologie (Terre et Vie), $\mathrm{n}^{\circ} 56$, pp. 373-387.

HIRZEL, A.H.; HAUSSER, J.; CHESSEL, D.; PERRIN N. (2002): «Ecological-niche factor analysis: how to compute habitat-suitability maps without absence data?». Ecology, nº 83, pp. 2027-2036.

KINZELBACH, R.; NICOLAI, B.; SCHLENKER, R. (1997): «Der Bienenfresser Merops apiaster als Klimazeiger: Zum Einflug in Bayern, der Schweiz und Baden im Jahr 1644». Journal für Ornithologie, no 138 (3), pp. 297-308.

KREBS, J.R.; AVERY, J. (1985): «Central Place Foraging in the European Bee-Eater, Merops apiaster». Journal of Animal Ecology, n 54(2), pp. 459-472.

MASSA, B.; RIZZO, M.C. (2002): «Nesting and feeding habits of the European Bee-eater (Merops apiaster L.) in a colony next to a beekeeping site». Avocetta, $\mathrm{n}^{\circ}$ 26, pp. 25-31.

MASSA, B.; LA MANTIA, T. (2007): «Forestry, pasture, agriculture and fauna correlated to recent changes in Sicily».Forest@, no 4, pp. 418-438. [online] URL: http://www. sisef.it/forest@/pdf/?id=efor0495-0040418 (con acceso el 17/05/2014).

MUNTANER, J.; FERRER, X.; MARTÍNEZ-VILALTA, A. (Eds.) (1983): Atlas dels ocells nidificants de Catalunya $i$ Andorra. Ketres Ed., Barcelona.

RATNIEKS, F.L.; CARRECK, N.L. (2010): «Clarity on honey bee collapse?». Science, $\mathrm{n}^{\mathrm{o}} 327$ (5962), pp. 152-153.

ROBLEDANO, F. (Dir.); FARINÓS, P.; ZAPATA, V.M.; SOTO, A.J. (2009): Programa de colaboración para el estudio de la presencia e impacto del abejaruco (Merops apiaster) en las explotaciones apícolas de la Región de Murcia ( $2^{a}$ fase). Asociación de Apicultores de la Región de Murcia y COAG-IR.

SÁNCHEZ-GUZMÁN, J.M. (Dir.). (2007): La evaluación del impacto de la ecología del Abejaruco (Merops apiaster) sobre el sector apícola en el ámbito rural transfronterizo de Extremadura. Consejería de Agricultura y Medio Ambiente. Junta de Extremadura.

SANTOS, X.; BRITO, J. C.; SILLERO, N.; PLEGUEZUELOS, J. M., LLORENTE, G.A.; FAHD, S.; PARELLADA, X. (2006): «Inferring habitat-suitability areas with ecological modelling techniques and GIS: A contribution to assess the conservation status of Vipera latastei». Biological Conservation, $\mathrm{n}^{\circ} 130$, pp. 416-425. 
SUNYER, C.; VIÑUELA, J. (1996): «Invernada de rapaces (O. Falconiformes) en España peninsular e Islas Baleares», en MUNTANER, J.; MAYOL, J. (Eds.): Biología y Conservación de las Rapaces Mediterráneas, Monografías, nº 4. SEO, Madrid, pp. 361-370. TELLERÍA, J.L. (1986): Manual para el censo de los vertebrados terrestres. Editorial Raíces, Madrid.

VALERA, F. (2014): «Abejaruco europeo - Merops apiaster», en Salvador, A., Morales, M. B. (Eds.): Enciclopedia Virtual de los Vertebrados Españoles. Museo Nacional de Ciencias Naturales, Madrid. [online] URL: http://www.vertebradosibericos.org/ (con acceso el 17/05/2014).

ZAPATA, V.; ROBLEDANO, F.; FARINÓS, P.; JIMÉNEZ, M.V. (2008): «Primeros datos acerca de la incidencia del abejaruco en los colmenares de la Región de Murcia». IV Jornada Técnica de Apicultura de la Región de Murcia, Moratalla. 